CLAWAR 2018: 21st International Conference on Climbing and Walking Robots and the Support Technologies for Mobile Machines, Panama City, Panama, 10-12 September 2018

\title{
A scalable, modular leg design for multi-legged stair climbing robots
}

\author{
T. BUETTNER* and D. WILKE and A. ROENNAU and G. HEPPNER \\ and R. DILLMANN \\ Intelligent Systems and Production Engineering (ISPE), \\ FZI Research Center for Information Technology, \\ Karlsruhe, 76131, Germany \\ *E-mail: timothee.buettner@fzi.de
}

\begin{abstract}
Improving robustness of walking robots has always been problematic. Their complex kinematics and locomotion has always been prone to damage: a broken cable, an unstable foothold or a wrong set of parameters has been an everlasting source of frustration. Nature developed an extraordinary robustness through redundancy and fast adaptation. Theories about decentralized nervous systems has inspired this paper with a novel approach. The presented solution aims at relocating low-level walking behaviours to a network of computers and, more exactly, into the robots individual legs. This paper will not cover the full scope of the software implementation (this is a field found especially in modular robotics), but presents how such an encapsulated leg with all necessary hardware is built and focuses on the mechanical and kinematic aspect of such legs. It highlights how a robotic leg needs to be designed to tackle structured environments serves as explanatory guide through the design process of legs with integrated PCU and sensors.
\end{abstract}

Keywords: Mechanical Design; Legged Locomotion; Assistive Robots; Walking Robots; Modular Robots.

\section{Introduction}

Insects and spiders have developed remarkable abilities of the course of their evolutionary history. Abilities that many researchers in the field of walking robots try to adapt and apply to their own systems. The most extreme behaviour such animals express is the voluntary amputation of endangered limbs. This autonomy is triggered in various situations and ranges from preventing venom to afflict the rest of the body ${ }^{1}$ to freeing itself from a fatal grip. $^{2}$ This skill became necessary due to the size of their bodies compared to their predators or dangers in their surroundings. The most remarkable skill though can be observed in the aftermath: the complete adaptation to 
the missing limb. Both represent inspiring qualities for robotics and are objective to many papers in the domain. The have been efforts in finding faster ways to recover from damage by exploring parameter spaces and avoiding diverging behaviours ${ }^{3}$ or make use of artificial neural networks to learn new walking strategies. ${ }^{4}$ The presented approach will more closely adapt the idea of decentralized behaviours. More exactly: it will propose a way of designing legs which house a decentralized computational unit for redundancy. A well known animal with such motion redundancy is the octopus. The behavioural and control hierarchy offers the capability to move limbs individually but overwrite signals to coordinate multi-limb movements (many controlled by individual brain lobes). ${ }^{5}$ Walking robots can't be compared
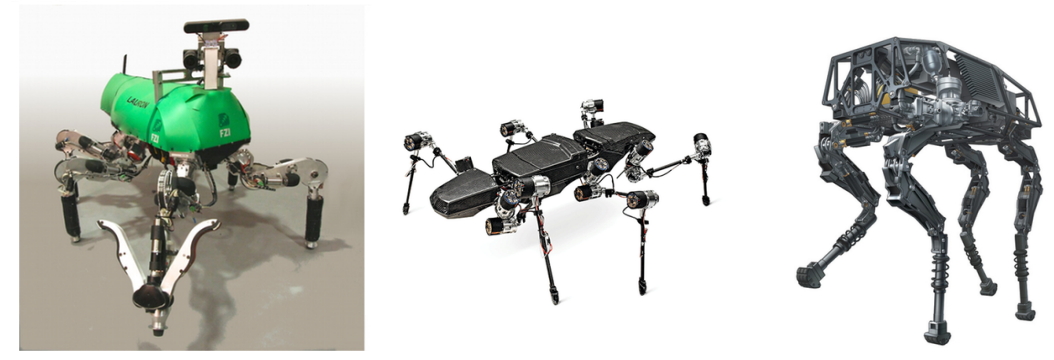

Fig. 1. Examples for different monolithic robot systems: (f.l.t.r.) LAURON V, ${ }^{6}$ HECTOR, ${ }^{7}$ BigDog. ${ }^{8}$

to this structure since their natural inspirations come from the domain of vertebrates or insects with different topologies (see Fig. 1). On the other hand, there has been efforts to recreate decentralized systems in walking robots by connecting different central pattern generators and recreating a motion control close to the intra-spinal neural network real animals ${ }^{9} .{ }^{10}$ This paper takes a mechanical approach to the problem. It will explain a way to design the basic leg kinematics for a walking robot to overcome obstacles met in daily life and also house enough internal computing power to be used as decentralized unit. Modularity, meaning the decoupling of the legs, will not be part of this work since this would cover another field and topic. The proposed design method will focus on the overarching development of a leg and the early assessment of possible obstacles. This is a more detailed design step and can be seen as a subroutine of more overarching design strategies as proposed by Tedeschi ${ }^{11}$ for example. The order in which each aspect of the robotic leg is presented also serves as possible procedure for other robotic leg designs. 


\section{Kinematic Restrictions}

Kinematic restrictions represent the minimum or maximum requirements a robotic leg has to meet to fulfil certain purposes. This can on one side be the workspace, length, carrying weight or the total mass for example. Each aspect defines the final shape of the robot. This section will take apart the design process and introduce a more accurate way of designing robotic legs by highlighting several pitfalls in this process. The leg was designed for a small walking robot with a minimum of six legs attached. The minimum weight to be carried was set to be approximately $2 \mathrm{~kg}$ with three degrees of freedom per leg (for a small demonstrator). The need for a smaller testing platform and the interest in decentralized walking is the driving factor for this small, intelligent leg. Using four DoF would allow the use of low energy walking strategies and intelligent posture control ${ }^{12}$ found in bigger robots such as LAURON V. ${ }^{6}$

\subsection{Robots and Environments}

The first step in defining the kinematic restrictions of a robot is assessment. The environment represents the biggest design factor right after the payload and motion type. Knowing the environment the robot will meet helps defining obstacles in its future path. As an example: The small decentralized demonstrator will be designed for indoor use. The ground is flat,solid and no dynamic changes occur. Nevertheless, there can be 2 more challenging obstacles involved: slopes and stairs. Other robotic systems will have to identify such obstacles in different environments, such as rocks, holes or bodies of water. With slopes being in place for wheelchair access and stairs as a general means of changing level within the building, this work will mainly focus on how to design the robot to climb these obstacles successfully. The following sections will explain, how these obstacles alter the shape of robots and require beforehand design decisions to render the robot capable of climbing successfully.

\subsection{Defining Proportions}

Starting top-down in design, the first decision is the number of joints per leg. These were set to be three, as explained beforehand, since redundancy or energy efficiency won't be part of this research. Starting from the joints, it is possible to define basic proportions of the leg without knowing the final length of it. Coming from an analogy to robotic manipulators, the work of Yoshikawa ${ }^{13}$ has shown a way of optimizing segment proportions by improv- 
ing its theoretical manipulability. This manipulability can be represented for non-redundant systems through (1).

$$
w=\|\operatorname{det} J(\Theta)\|
$$

with (2) representing the vector of joint angles

$$
\Theta=\left[\Theta_{1}, \Theta_{2}, \Theta_{3}, \ldots, \Theta_{m}\right]^{T}
$$

and (3) its Jacobian.

$$
J(\theta) \in R^{m \times n}
$$

By decomposition of the Jacobian into its singularities $\sigma_{1}, \sigma_{2}, \sigma_{3}, \ldots \sigma_{m}$, the manipulability becomes

$$
w=\sigma_{1} \cdot \sigma_{2} \cdot \sigma_{3} \cdot \ldots \cdot \sigma_{m}
$$

Expressed in the terms of a 3DOF Robot with $L_{1}, L_{2}, L_{3}$ being its segment lengths and $\Theta_{1}, \Theta_{2}, \Theta_{3}$ being its joint angles, formula (4) becomes

$$
w=L_{2} \cdot L_{3} \cdot\left|\left(L_{2} \cdot \sin \left(\Theta_{2}\right)+L_{3} \cdot \sin \left(\Theta_{2}+\Theta_{3}\right)\right) \cdot \sin \left(\Theta_{3}\right)\right|
$$

This formula has its maximum for $L_{2}=L_{3}$ and $\Theta_{3}= \pm 90^{\circ}$. The manipulability can be described as the capacity of the manipulator to change the orientation and position of its end-effector at a given joint state. But since the robotic leg moves freely, the $90^{\circ}$ angles can't be maintained. Still, it is possible to use the proportions $L_{2}=L_{3}$ as design restrictions for the two longest segments: lower and upper leg. Even though climbing is not considered a manipulation task, applying this rule leads to a larger manipulation volume, allowing more variety in footpoints.

\subsection{Minimum Length}

Returning to the environmental restrictions, there are several leg parameters that can be calculated from known obstacle sizes. The stairs have been identified as the most challenging obstacles in an indoor environment as many multi-legged robots will fail if their legs are not scaled correctly. The more prominent examples for stair climbing robots are BigDog from Boston Dynamics or ANYmal from ETH Zurich. ${ }^{14}$ Six and more legged examples have only vaguely shown success. One example being the modular robot Snake Monster from Carnegie Mellon. The comparison of both climbing styles shows how four legged approaches exceed their six legged competitors: The leg constellation is rotated in a way, that most of the 
workspace covers the stair area (similar to mammalian topologies). In comparison, insect-like arrangements allow a better foothold but under-perform if bodies have to be pushed upwards and forwards. For the next step, we will assume the robotic legs will be placed parallel to the sagittal plane. Selecting the body pose - Even though most stairs have regulatory obligations to remain within a certain range of height (this also counts for slopes) no works have been made to link the design process directly to these rule sets to define robotic lengths. We are looking at the case with the least favourable body orientation: The robot body is in direct contact with the stairs. This orientation can either be parallel to the stair inclination or horizontal (as sketched in Fig. 2).

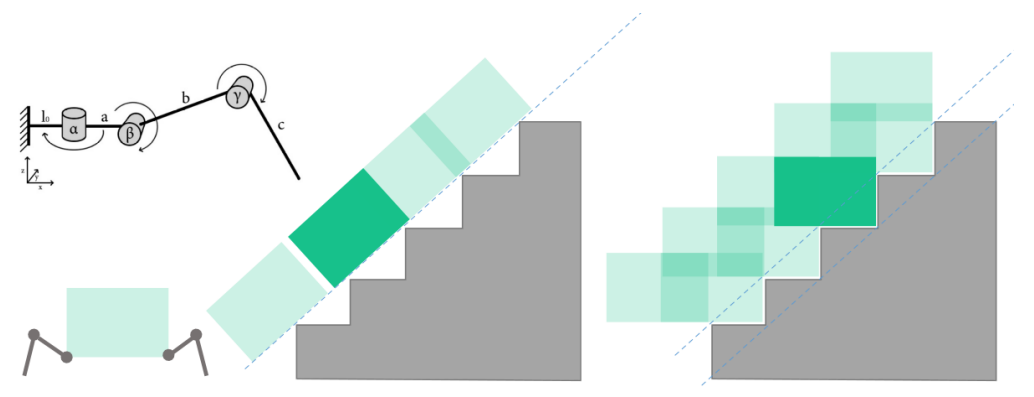

Fig. 2. Two possible body poses for stair climbing robots with sagittal leg constellations. The simplified image shows the closest positions in contact with the stair outline. The tilted approach is found more often than a constant horizontal position (r.).

Maximum and minimum inclination - Stairs have strict regulations which allows the estimation of possible obstacles for indoor environments. There are several international rules that differ slightly. The regulation for this example is drawn from the German DIN 18065. The indoor riser height lies between 140 and $200 \mathrm{~mm}$ and the tread depth between 230 to $370 \mathrm{~mm}$. As an example, the International Residential Code for Stair treads and risers R311.5.3 has a maximum riser height of $196 \mathrm{~mm}$ and minimum tread depth of $254 \mathrm{~mm}$. These numbers describe the majority of stairs in buildings. The maximum and minimum slope a robot can encounter are calculated by combining the highest rise with the shortest tread (and vice versa). The resulting slopes can be seen in Fig. 3.The maximum slope will be taken as design reference since it highlights the steepest obstacle for an indoor robot. As a result. All calculations will be made with the inclination of $41^{\circ}$, a riser height of $200 \mathrm{~mm}$ and a tread depth of $230 \mathrm{~mm}$. 


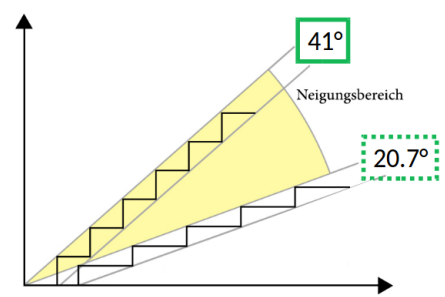

Fig. 3. Relative comparison of the highest and lowest slope angle. Both are drawn from the values found in DIN 18065.

Minimum length - Next would be the calculation of the minimum leg length through geometrical approximation. This is done by selecting the closest possible distance a horizontally oriented robot would take while it is climbing stairs. This minimum distance is sketched in Fig. 4: The robot has its front and bottom pressed against the stairs and is therefore closest to the stairs. Any other position would require longer legs. To move the robot forward, the following order of movement is required:

(1) The robot in Fig. 4 is currently at the beginning of the step. The hind legs are extended and the front legs are pushed against the stair.

(2) The robot needs to move its hind legs one step up to push its body upwards alongside the stair.

(3) The hind leg then pushes the robot forward to maintain minimum distance with the stair.

(4) The new position is one stair further but in the same constellation.

Fig. 4 also shows, that any downward movement of the extended hind leg is impossible since it would collide with the edge of the stairs. This occurrence also represents the geometric connection between the shortest robotic leg and the size of the stairs: It is impossible for robots with a body length shorter than the stair tread (in this example $230 \mathrm{~mm}$ ) to shorten their leg any further. The robot wouldn't be able to lift its body to the next step in a single movement. This minimum length necessary to lift the robot up one stair at the time represents a measure that is independent from any speed or gait since it maintains the smallest possible distance between robot and stairs. Using the two tread depths of $460 \mathrm{~mm}$, riser heights of $400 \mathrm{~mm}$ and the $41^{\circ}$ slope, the length results to $l_{\text {total }}=609 \mathrm{~mm}$ from shoulder joint to foot.

Longer robots - Surely it is possible to apply this to longer robots, in which case legs become shorter as the robot grows in length. The greyed out sketch 
in Fig. 4 shows the effect of an extended body length: The robot is able to move the leg lower (compared to the short version), thus allowing him to push itself to the next step while having its footpoints move inwards. A robot with $400 \mathrm{~mm}$ body length would only require $l_{\text {total }}=418 \mathrm{~mm}$ in total length at its hind legs. The absolute minimum would be reached with a robot length of twice the tread with the shortest distance to the step being twice the step $\left(l_{\min }=400 \mathrm{~mm}\right)$.

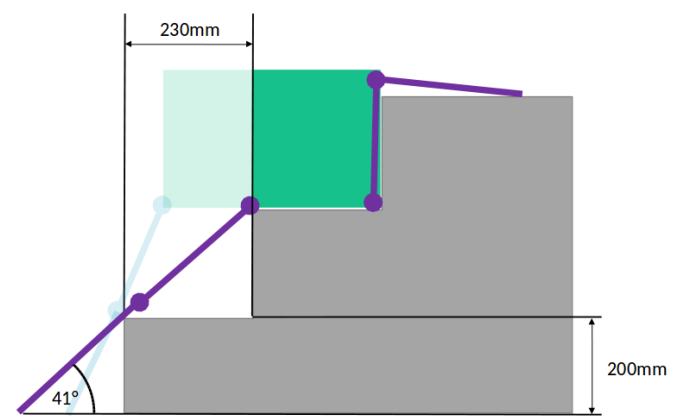

Fig. 4. Relative comparison of the highest and lowest slope angle. Both are drawn from the values found in DIN 18065.

\section{Mechanical Design}

The mechanical design was derived from the beforehand explained kinematic restrictions (proportions and length). The subsequent step would be the theoretical load cases for such a climbing robot. Working with the extreme load-cases for walking robots, one would assume that extending the robots legs away from its body is the classic maximum: the distance between footpoints and centre of mass is a full leg-length apart which causes the maximum torque in the beta joint. To take a different approach and because it is unlikely the robot might get into this situation, a different, more probable load-case was selected. Fig. 5 shows the case for stair climbing: The robots CoM is vertically aligned with one row of footpoints. This result the entire load being carried by only these legs. Placing the legs orthogonally to the gravitational force results in the largest possible torque (in this case for joint $\beta$ )

The resulting torque (with the center of mass placed above one leg as in Fig. 5) can be expressed as follows:

$$
T_{\text {max }}=m_{\text {total }} \cdot g \cdot L_{\text {segment }}
$$




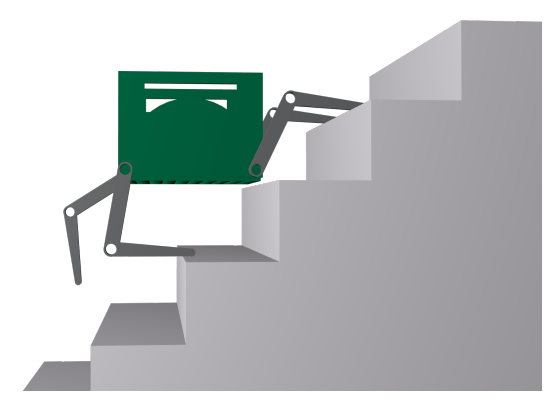

Fig. 5. A highly unfavourable pose: the centre of mass is aligned directly above the footpoints while the lower limb itself is arranged orthogonally.

Having finished the theoretical part of the calculations, one would need to add a first estimate of the robots mass to the equation (6). This is necessary and part of every design process: later calculations or materials will most like (and as in our case) change the final numbers the torque values. We have picked an estimated mass of $m_{\text {total }}=15 \mathrm{~kg}$ as initial guess from experience. This step may vary depending on the planned robotic setup, payload and material. Adding this to the equation results in $T_{\max }=$ $32.373 \mathrm{Nm}$ (for our segment length of $0.22 \mathrm{~m}$ ).

\subsection{Structure}

The structure was conceived to be capable of housing an IMU, and gyroscope in its shoulder as well as the motors with their corresponding transmission. The theoretical proportions were integrated with both $\beta$ and $\gamma$ shafts being $0.22 \mathrm{~m}$ apart and both leg segments being equidistant. The casing as seen in Fig. 6 was designed to be milled from aluminium. Extensive load calculations and computer aided analysis have lead to the decision to manufacture both drive shafts of $\beta$ and $\gamma$ separately since aluminium was unfit for most load-cases.

\section{Conclusion and Future Works}

A new concept for an decentralized robotic leg was presented in detail. With focus on the theoretical proportions of the leg, all steps of the design process were highlighted. Starting with the definition of proportions, which were calculated via the Yoshikawa ellipsoids, the leg was designed to be biologically inspired. The length was derived from the robots environmental restrictions and planned stair climbing. A novel method of calculating the 


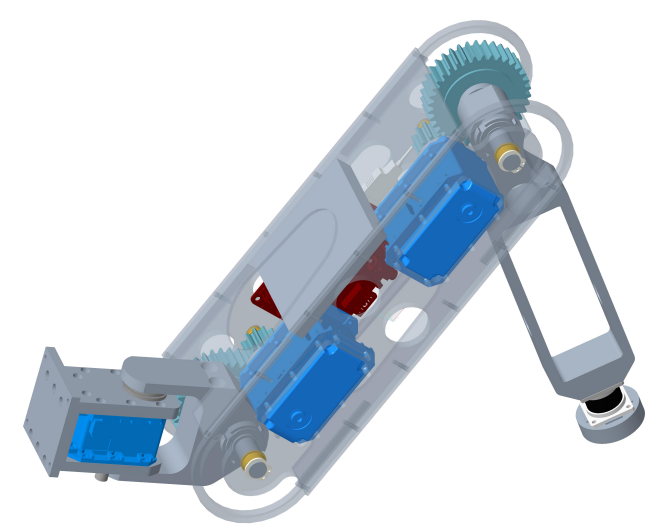

Fig. 6. A complete view of the robotic leg. The blue motors represent MX-106 model actuators. The dark red part is an integrated Raspberry Pi 3 for decentralized task handling.

minimum required leg length was proposed as well. The method links the robots legs length to the geometric values of stairs and can be applied for any robot with such climbing tasks. Future works can mainly be seen in the development of a more detailed formula for stair climbing. The presented use-case only covers a part of the robots possibilities and needs further elaboration. Using this concept on rocky terrain is planned in the future but requires geometric abstraction of the terrain. Such design formula would allow the calculation of the optimum length of legs with the knowledge of the terrain characteristics. Tests are planned with the first leg design (shown in Fig.7).

\section{References}

1. T. Eisner and S. Camazine, Spider leg autotomy induced by prey venom injection: An adaptive response to 'pain'? (1983).

2. P. A. Fleming and P. W. Bateman, Just drop it and run: the effect of limb autotomy on running distance and locomotion energetics of field crickets (gryllus bimaculatus) (2007).

3. S. Koos, A. Cully and J.-B. Mouret, Fast damage recovery in robotics with the t-resilience algorithm (2013).

4. D. Berenson, N. Estevez and H. Lipson, Hardware evolution of analog circuits for in-situ robotic fault-recovery, in 2005 NASA/DoD Conference on Evolvable Hardware (EH'05), June 2005.

5. L. Zullo, G. Sumbre, C. Agnisola, T. Flash and B. Hochner, Nonsomatotopic organization of the higher motor centers in octopus (2009). 


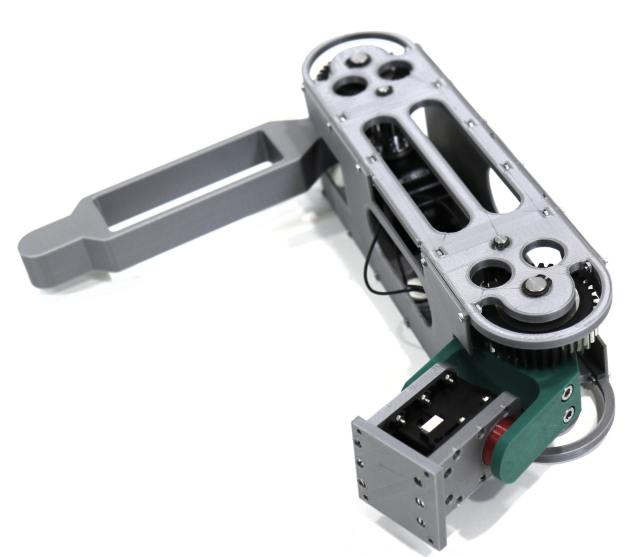

Fig. 7. The final manufactured leg. The initial aluminium parts were manufactured in FDM (PLA base) to reduce weight furthermore.

6. A. Roennau, G. Heppner, M. Nowicki and R. Dillmann, Lauron v: A versatile six-legged walking robot with advanced maneuverability(07 2014).

7. A. Schneider, J. Paskarbeit, M. Schaeffersmann and J. Schmitz, Hector, a new hexapod robot platform with increased mobility - control approach, design and communication, in Advances in Autonomous Mini Robots, eds. U. Rückert, S. Joaquin and W. Felix (Springer Berlin Heidelberg, Berlin, Heidelberg, 2012).

8. D. Wooden, M. Malchano, K. Blankespoor, A. Howardy, A. A. Rizzi and M. Raibert, Autonomous navigation for bigdog, in 2010 IEEE International Conference on Robotics and Automation, 2010.

9. D. Owaki, T. Kano, K. Nagasawa, A. Tero and A. Ishiguro, Simple robot suggests physical interlimb communication is essential for quadruped walking(10 2012).

10. E. Berg, S. Hooper, J. Schmidt and A. Bschges, A leg-local neural mechanism mediates the decision to search in stick insects(07 2015).

11. F. Tedeschi and G. Carbone, 3, 181(06 2014).

12. A. Roennau, G. Heppner, M. Nowicki, J. Zoellner and R. Dillmann, Reactive posture behaviors for stable legged locomotion over steep inclines and large obstacles(09 2014).

13. T. Yoshikawa, Manipulability and redundancy control of robotic mechanisms, in Proceedings. 1985 IEEE International Conference on Robotics and Automation, Mar 1985.

14. T. Takuma and W. Kase, Robust and directive quadruped locomotion on rough terrain without requiring sensing and actuation, in 2016 IEEE International Conference on Robotics and Biomimetics (ROBIO), Dec 2016.

15. S. Kalouche, Snake monster stair ascent https://www.youtube.com/watch?v=urjp3wb3vsy. 